\title{
FABER SERIES AND THE LAURENT DECOMPOSITION
}

\author{
H. Tietz
}

\section{INTRODUCTION}

This paper deals with the problem of transfer described by J. L. Ullman [5]. Roughly speaking, we should like to have a method for deciding what statements about a Faber series

$$
\sum_{0}^{\infty} a_{n} F_{n}(z)
$$

are equivalent to analogous statements about the associated power series

$$
\sum_{0}^{\infty} a_{n} z^{n}
$$

with the same coefficients.

Ullman partially solved the problem by means of a lemma concerning the rationality of functions: if one of the series (1) and (2) represents a rational function, then the same is true of the other. The lemma leads to an immediate proof, for example, of Iliev's analogue [2] of Szëgo's theorem on power series whose coefficients assume only a finite number of different values.

We shall establish a result (Theorem 2) which is at the same time more elementary and more general than Ullman's lemma. It asserts that if $f(z)$ denotes the mapping function, normalized at $\mathrm{z}=\infty$, which is associated with the analytic curve $\mathrm{C}$ giving rise to the Faber sequence $\left\{F_{n}(z)\right\}$, then the difference between the series (1) and the series

$$
\sum_{0}^{\infty} a_{n}[f(z)]^{n}
$$

can be continued so as to be holomorphic everywhere on $\mathrm{C}$ and outside of $\mathrm{C}$. The proof of this theorem is based on a very simple tool, the Laurent decomposition. This device (developed by the author in connection with the extension of the Faber theory to Riemann surfaces; see [3], [4]) is described in Section 2. In Section 3, we give a brief development of the Faber theory, and we prove our fundamental result. The final section is devoted to applications of the theorem. 


\section{THE LAURENT DECOMPOSITION}

2.1. Let $C$ be a rectifiable Jordan curve in the $z$-plane, with the interior $I(C)$ and the exterior $A(C)$. With every (single-valued) function $\phi$ holomorphic on $C$ we associate the two functions

$$
\begin{aligned}
& L \phi(z)=\frac{1}{2 \pi i} \int_{C} \phi(\zeta) \frac{d \zeta}{\zeta-z} \quad \text { for } z \in I(C), \\
& L^{*} \phi(z)=\frac{-1}{2 \pi i} \int_{C} \phi(\zeta) \frac{d \zeta}{\zeta-z} \quad \text { for } z \in A(C) .
\end{aligned}
$$

Since the path of integration may be moved slightly, in the neighborhood of the curve $C$, we see that $L \phi$ is holomorphic in $I(C) \cup C$, and that $L^{*} \phi$ is holomorphic in $A(C) \cup C$, with $L^{*} \phi(\infty)=0$. Therefore $L \phi$ and $L^{*} \phi$ are holomorphic on $C$, and

$$
\phi=L \phi+L * \phi
$$

on $C$. In the special case where $C$ is a circle, this relation reduces to the wellknown Laurent decomposition of a function which is holomorphic in an annulus containing $\mathrm{C}$. We therefore call $\mathrm{L}$ and $\mathrm{L}^{*}$ the operators of the Laurent decomposition with respect to $C$. that is,

2.2. From (4) it follows immediately that the operator $L$ is linear and continuous;

$$
L(a \phi+b \psi)=a L \phi+b L \psi
$$

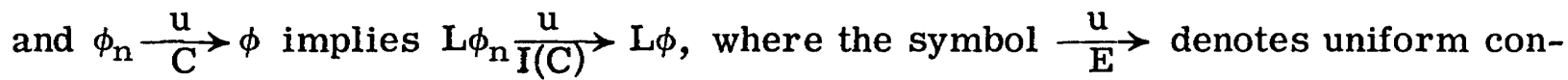
vergence on every compact subset of $\mathrm{E}$. In particular, if $\phi=\mathrm{F}$ is holomorphic in $I(C) \cup C$, then $L F=F$, and therefore

$$
\phi_{\mathrm{n}} \underset{\mathrm{C}}{\stackrel{\mathrm{u}}{\longrightarrow}} \mathbf{F}
$$

implies immediately

$$
\mathrm{L} \phi_{\mathrm{n}} \underset{\mathrm{I}(\mathrm{C})}{\rightarrow} \mathrm{F} .
$$

\section{FABER THEORY}

3.1. Henceforth, we assume that the curve $C$ is analytic. Let $t=f(z)$ be a conformal mapping of $A(C)$ onto the exterior of the circle $K:\{|t|=k\}$, with $f(\infty)=\infty$. Clearly, $f$ is holomorphic and schlicht also on $\mathrm{C}$.

Now let $F$ be a function which is holomorphic in $I(C) \cup C$; then the mapping $f$ carries $F$ into a function $G$ which is holomorphic on $K$ and which has a Laurent expansion 


$$
G(t)=\sum_{-\infty}^{\infty} a_{n} t^{n}
$$

on $\mathrm{K}$. Interpreting this series in terms of values $\mathrm{z}$ on $\mathrm{C}$ and in the neighborhood of $\mathrm{C}$, we deduce that

$$
F(z)=\sum_{-\infty}^{\infty} a_{n}[f(z)]^{n}
$$

on C; we shall call (10) a Laurent series. By (7), (8) and (9), it follows from (10) that

$$
F(z)=\sum_{-\infty}^{\infty} a_{n} L[f(z)]^{n} \quad(z \in I(C))
$$

3.2. By (6), the relation $\mathrm{f}^{\mathrm{n}}=\mathrm{L} \mathrm{f}^{\mathrm{n}}+\mathrm{L} *_{\mathrm{f}^{n}}$ holds throughout the neighborhood of $\mathrm{C}$.

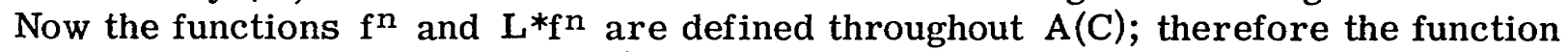
$\mathrm{Lf}^{\mathrm{n}}$, which is holomorphic in $\mathrm{I}(\mathrm{C})$, can be continued into the entire plane, and

$$
\mathrm{Lf}^{n}=\mathrm{f}^{\mathrm{n}}-\mathbf{L} *_{\mathrm{f}} \mathrm{n}
$$

throughout $A(C)$. The second term on the right is holomorphic in $A(C)$ and vanishes at $\mathrm{z}=\infty$. Since the function $\mathrm{f}^{\mathrm{n}}$ also vanishes at $\mathrm{z}=\infty$, when $\mathrm{n}<0$, it follows that

$$
\mathrm{Lf}^{\mathrm{n}} \equiv 0 \quad(\mathrm{n}<0)
$$

On the other hand, for $\mathrm{n} \geq 0$ the function $\mathrm{f}^{\mathrm{n}}$ is holomorphic in $\mathrm{A}(\mathrm{C})$ except for a pole of multiplicity $n$ at $z=\infty$. It follows that $L_{\mathbf{f}^{n}}$ is a polynomial of degree $n$; this polynomial is called the Faber polynomial $\mathrm{F}_{\mathrm{n}}$ (relative to the curve $\mathrm{C}$ ):

$$
\mathrm{Lf}^{\mathrm{n}} \equiv \mathrm{F}_{\mathrm{n}} \quad(\mathrm{n} \geq 0) .
$$

Returning to the function $F$ discussed in Section 3.1, we note that because the curve $\mathrm{C}$ is analytic, the hypothesis that $\mathrm{F}$ is holomorphic on $\mathrm{C}$ can be dropped: if $F$ is holomorphic in $I(C)$, we can replace the curve $C$ by a curve $C^{\prime}$ which lies in $I(C)$ and which is sufficiently near to $C$ so that $f$ is also holomorphic throughout $A\left(C^{\prime}\right) \cup C^{\prime}$. Nothing is changed, then, when we refer the operators $L$ and $L^{*}$ to the curve $C^{\prime}$ instead of to $C$. From (11), (12) and (13) we now obtain the following classical result:

THEOREM 1. If the function $\mathrm{F}$ is holomorphic in $\mathrm{I}(\mathrm{C})$, it can be represented by a Laurent expansion $\mathrm{F}=\Sigma_{-\infty}^{\infty} \mathrm{a}_{\mathrm{n}} \mathrm{f}^{\mathrm{n}}$ near $\mathrm{C}$ and by a Faber expansion $\mathrm{F}=\Sigma_{0}^{\infty} \mathrm{a}_{\mathrm{n}} \mathrm{F}_{\mathrm{n}}$ in $\mathrm{I}(\mathrm{C})$.

3.3. If a series (1) converges uniformly in each compact subset of $I(C)$, we call it a Faber series. Let $F$ denote the holomorphic function to which such a series converges. If we replace each of the functions $F_{n}$ by its Laurent series in $f$ and note that this "series" consists of precisely two terms, we obtain the relation

$$
F=\sum_{-\infty}^{\infty} b_{n} f^{n}
$$


where $b_{n}=a_{n}$ for $n \geq 0$. Since the Laurent coefficients are uniquely determined by $F$, the Faber series of the function is also uniquely determined. And since the function $\Sigma_{-\infty}^{-1} b_{n} f^{n}$ is holomorphic in $A(C) \cup C$ and vanishes at $z=\infty$, we have established the following conclusion.

THEOREM 2. Let $\Sigma_{0}^{\infty} a_{n} F_{n}$ be a Faber series, let $\mathrm{F}(\mathrm{z})$ denote its sum in $\mathrm{I}(\mathrm{C})$, and let $\mathrm{g}(\mathrm{z})=\Sigma_{0}^{\infty} \mathrm{a}_{\mathrm{n}} \mathrm{f}^{\mathrm{n}}$. Then the function $\mathrm{F}(\mathrm{z})-\mathrm{g}(\mathrm{z})$ can be continued analytically throughout $\mathrm{A}(\mathrm{C}) \cup \mathrm{C}$; it is represented there by the series $\Sigma_{0}^{\infty} \mathrm{a}_{\mathrm{n}}\left(\mathrm{F}_{\mathrm{n}}-\mathrm{f}^{\mathrm{n}}\right)$; and it vanishes at $\mathrm{z}=\infty$.

COROLLARY். Let $\Sigma \mathrm{a}_{\mathrm{n}} \mathrm{F}_{\mathrm{n}}$ be a Faber series, and let $\mathrm{R}_{1}$ and $\mathrm{R}_{2}$ denote the Riemann surfaces on which the two functions $\Sigma_{0}^{\infty} \mathrm{a}_{\mathrm{n}} \mathrm{F}_{\mathrm{n}}$ and $\Sigma_{0}^{\infty} \mathrm{a}_{\mathrm{n}} \mathrm{f}^{\mathrm{n}}$ are holomorphic. Then the portions of $\mathrm{R}_{1}$ and $\mathrm{R}_{2}$ which lie above the set $\mathrm{A}(\mathrm{C}) \cup \mathrm{C}$ are identical.

In particular, Ullman's lemma becomes immediate if we interpret the "power series" $\Sigma_{0}^{\infty} a_{n} f^{n}$ as the series $\Sigma_{0}^{\infty} a_{n} t^{n}$.

\section{FABER SERIES}

4.1. It follows from the proof of Theorem 2 that a Faber series $\Sigma_{0}^{\infty} a_{n} F_{n}$ and its associated power series $\Sigma_{0}^{\infty} a_{n} f^{n}$ have a common "ring" of convergence in $I(C)$. Since the operator $L^{*}$, like $L$, is linear and continuous, it follows that in $A(C) \cup C$ the partial sums $s_{n}^{*}=\Sigma_{0}^{n} a_{k} L^{*}{ }^{k}$ converge uniformly to a holomorphic function. By (6) and (13), the corresponding partial sums

$$
\sigma_{n}=\sum_{0}^{n} a_{k} F_{k} \quad \text { and } \quad s_{n}=\sum_{0}^{n} a_{k} f^{k}
$$

satisfy the condition $\sigma_{n}=s_{n}-s_{n}^{*}$, on $A(C) \cup C$, and it follows that if one of two sequences $\left\{\sigma_{n_{i}}\right\}$ and $\left\{s_{n_{i}}\right\}$ converges (uniformly) on some subset of $A(C) \cup C$, then the other does likewise, and the difference of the two limits can be extended so that it is holomorphic in $A(C) \cup C$. We summarize:

THEOREM 3. The series $\Sigma \mathrm{a}_{n} \mathrm{~F}_{\mathrm{n}}$ and $\Sigma \mathrm{a}_{\mathrm{n}} \mathrm{f}^{\mathrm{n}}$ have the same sets of convergence and of uniform convergence, in $\mathrm{A}(\mathrm{C}) \cup \mathrm{C}$. The same applies to sets of overconvergence, natural boundaries, and sets of continuity at the boundary.

More can be said; but it is obviously not feasible to compile a catalogue of theorems on Taylor series which can be restated in terms of Faber series.

4.2. Finally, we deduce a result of $P$. Heuser [1], by the method of the Laurent decomposition. Let $C_{i}(i=1,2)$ be an analytic simple closed curve in the $z_{i}$-plane, and let the functions $t=f_{i}\left(z_{i}\right)$ map the domains $A\left(C_{i}\right)$ conformally onto the exterior of the same circle $K$ in the $t$-plane. Let $F_{h}^{(i)}\left(z_{i}\right)$ and $L_{i}$ denote the corresponding Faber polynomials and Laurent operators. We wish to find a relation between functions

$$
F^{(1)}\left(z_{1}\right)=\sum_{0}^{\infty} a_{n} F_{n}^{(1)}\left(z_{1}\right) \quad \text { and } \quad F^{(2)}\left(z_{2}\right)=\sum_{0}^{\infty} a_{n} F_{n}^{(2)}\left(z_{2}\right)
$$

with the same set of Faber coefficients. 
Let

$$
F^{(1)}\left(z_{1}\right)=\sum_{-\infty}^{\infty} a_{n}\left[f_{1}\left(z_{1}\right)\right]^{n}
$$

be the Laurent expansion of $F^{(1)}\left(z_{1}\right)$ near $C_{1}$. Since the relation $t=f_{1}\left(z_{1}\right)=f_{2}\left(z_{2}\right)$ defines a holomorphic mapping $z_{1}=\gamma\left(z_{2}\right)$ of $A\left(C_{2}\right) \cup C_{2}$ onto $A\left(C_{1}\right) \cup C_{1}$, the relation

$$
F^{(1)}\left(\gamma\left(z_{2}\right)\right)=\sum_{-\infty}^{\infty} a_{n}\left[f_{2}\left(z_{2}\right)\right]^{n}
$$

holds near $\mathrm{C}_{2}$. On the other hand,

$$
L_{2}\left(\sum_{-\infty}^{\infty} a_{n}\left[f_{2}\left(z_{2}\right)\right]^{n}\right)=\sum_{0}^{\infty} a_{n} F_{n}^{(2)}\left(z_{2}\right)=F^{(2)}\left(z_{2}\right)
$$

From this follows Heuser's result:

$$
F^{(2)}\left(z_{2}\right)=L_{2}\left(F^{(1)}\left[\gamma\left(z_{2}\right)\right]\right)=\frac{1}{2 \pi i} \int_{C_{2}} F^{(1)}[\gamma(\zeta)] \frac{d \zeta}{\zeta-z_{2}} \quad\left(z_{2} \in I\left(C_{2}\right)\right)
$$

\section{REFERENCES}

1. P. Heuser, Ueber eine Transformation der Faberschen Polynomreihen, Math. Z. 38 (1934), 777-782.

2. L. Iliev, Series of Faber polynomials whose coefficients assume a finite number of values, Doklady Akad. Nauk SSSR (N.S.) 90 (1953), 499-502.

3. H. Tietz, Laurent-Trennung und zweifach unendliche Faber Systeme, Math. Ann. 129 (1955), 431-450.

4. - Faber-Theorie auf nicht-kompakten Riemannschen Flächen, Math. Ann. 132 (1957), 412-429.

5. J. L. Ullman, On Faber series. 1. A problem of transfer, Michigan Math. J. 2 (1953-1954), 109-114.

University of Münster, Westphalia, Germany 


\section{,}

